\title{
Serum gamma glutamyl transferase in patients with sleep apnea-hypopnea
}

\author{
Tomoyuki Kawada ${ }^{1}$
}

Received: 25 March 2015 / Accepted: 16 June 2015 /Published online: 9 July 2015

(C) Springer-Verlag Berlin Heidelberg 2015

\section{Dear Editor:}

Sanchez-Armengol et al. reported the association between serum gamma glutamyl transferase (GGT) and sleep apneahypopnea in combination with alcohol consumption and several metabolic factors including obesity [1]. The authors concluded that high GGT was mainly associated with central obesity, rather than sleep apnea-hypopnea by multivariate analysis. I have some concerns on their study outcome.

Firstly, the authors handled 1744 patients (1360 men and 384 women) with suspected symptoms of sleep apneahypopnea. I suppose that there are patients who never drink, but the authors calculated the level of alcohol consumption of all the patients as grams per day according to the simple formula. As this indicator was used for univariate and multivariate analysis, statistical results should be handled with caution (please see the big standard deviations against mean values). I recommend using the level of alcohol consumption as categorical data as it is not normally distributed.

Secondly, the authors should check data of multivariate logistic regression mode in their Table 4. I suppose that $95 \%$ confidence intervals are not described for odds ratios (ORs). As these values cannot be considered as logarithmic values of ORs, please specify the meaning of the values. In addition, the authors inserted three independent variables of binary apnea-hypopnea index (AHI) in combination with continuous value of AHI. I recommend not using continuous AHI

Tomoyuki Kawada

kawada@nms.ac.jp

1 Department of Hygiene and Public Health, Nippon Medical School, 1-1-5 Sendagi, Bunkyo-Ku, Tokyo 113-8602, Japan and categorical AHI simultaneously as independent variables for GGT levels $\geq 40 \mathrm{IU} / 1$. In case of categorical AHI indices, please use standard classification, which was described in their "Material and methods" section as mild, moderate, and severe. Furthermore, the authors described OR of waist-hip ratio for GGT levels $\geq 40 \mathrm{IU} / 1$ being 21.5 , and standard error of waist-hip ratio was very big, presenting unstable estimate of OR. Instead of absolute value of OR, the level of statistical significance should be adopted for the association with high GGT level.

Finally, I suppose that overnight sleep study was conducted for subjects without alcohol consumption at that night even for drinkers. The acute effect of alcohol consumption on subsequent sleep-disordered breathing has been reported [2]. Prevention of heavy drinking to prevent sleep apneahypopnea and associated diseases for drinker should be considered important [3].

Disclosure statement The author has indicated no financial support.

Conflict of interest The author declares no competing interests.

\section{References}

1. Sanchez-Armengol A, Villalobos-Lopez P, Caballero-Eraso C, Carmona-Bernal C, Asensio-Cruz M, Barbe F, Capote F (2015) Gamma glutamyl transferase and oxidative stress in obstructive sleep apnea: a study in 1744 patients. Sleep Breath. doi:10.1007/s11325014-1115-5

2. Stein MD, Friedmann PD (2005) Disturbed sleep and its relationship to alcohol use. Subst Abus 26(1):1-13

3. Heinzer R, Vat S, Marques-Vidal P, Marti-Soler H, Andries D, Tobback N, Mooser V, Preisig M, Malhotra A, Waeber G, Vollenweider P, Tafti M, Haba-Rubio J (2015) Prevalence of sleepdisordered breathing in the general population: the HypnoLaus study. Lancet Respir Med 3(4):310-318 